University of San Diego

Digital USD

Spring 5-22-2021

\title{
Motivational Interviewing and Frequent Follow-up in a Pediatric Primary Care Setting to Improve Diet and Activity in Adolescents
}

Celeste Armenta

University of San Diego, celestearmenta@sandiego.edu

Kathy James

University of San Diego, kjames@sandiego.edu

Follow this and additional works at: https://digital.sandiego.edu/dnp

Part of the Nursing Commons

\section{Digital USD Citation}

Armenta, Celeste and James, Kathy, "Motivational Interviewing and Frequent Follow-up in a Pediatric Primary Care Setting to Improve Diet and Activity in Adolescents" (2021). Doctor of Nursing Practice Final Manuscripts. 164.

https://digital.sandiego.edu/dnp/164

This Doctor of Nursing Practice Final Manuscript is brought to you for free and open access by the Theses and Dissertations at Digital USD. It has been accepted for inclusion in Doctor of Nursing Practice Final Manuscripts by an authorized administrator of Digital USD. For more information, please contact digital@sandiego.edu. 


\title{
UNIVERSITY OF SAN DIEGO
}

Hahn School of Nursing and Health Science DOCTOR OF NURSING PRACTICE

Motivational Interviewing and Frequent Follow-up in a Pediatric Primary Care Setting to Improve Diet and Activity in Adolescents

by

Celeste Armenta, RN, FNP-BC

A Doctor of Nursing Practice Portfolio presented to the FACULTY OF THE HAHN SCHOOL OF NURSING AND HEALTH SCIENCE UNIVERSITY OF SAN DIEGO

\author{
In partial fulfillment of the \\ Requirements for the degree \\ DOCTOR OF NURSING PRACTICE
}

May 2021

Kathy James, DNSc, ARNP, FAAN, Faculty Advisor 


\begin{abstract}
Background: Adolescent obesity is a serious health concern with increased worldwide prevalence. Excess weight gain can affect adolescent's physical and emotional health. The adolescent with excess weight can involve acute and chronic long-term health complications that continue into adulthood.

Methods: Health care providers screened for overweight and obese adolescents during annual physicals and follow-up visits. Thirty adolescents at the 85 th - 95th percentile and above on the growth chart received a pre and post clinical-screening questionnaire for eating behaviors and exercise. Individualized education with parent involvement and motivational interviewing (MI) was implemented with monthly follow-ups for 4 months.
\end{abstract}

Findings: Data were collected over 4 months. A modified clinical-screening questionnaire for eating behaviors revealed improvements in eating habits, exercise, and a 5\% overall average weight loss as measured by BMI. As healthy behaviors increased, BMI decreased $(r=-.75, p<$ $.001)$. As the number of visits increased, healthy behaviors increased $(r=.79, \mathrm{p}<.001)$.

Discussion: The use of MI with guided behavioral modifications by practitioners with parent involvement and frequent follow-up can improve healthy behaviors and exercise in the adolescents.

Implications for Practice: This project supports that MI with guided behavioral changes by practitioner and parent involvement improves adolescents' diet and physical activity. MI and frequent follow-up were effective in promoting positive behavior change and healthier lifestyle changes.

Key Words: adolescents, clinical screening questionnaire, motivational interviewing, parent involvement, healthy behaviors 
Motivational Interviewing and Frequent Follow-up in a Pediatric Primary Care Setting to Improve Diet and Activity in Adolescents

\section{Background}

Adolescent obesity has become a significant problem in the United States. According to the Centers for Disease Control (Hales et al., 2017), obesity prevalence continues to rise and is at 20.6\% among adolescents between the ages of 12 and 19 years. Adolescent obesity contributed to weight-related diseases, affecting one's health throughout adulthood (Ayala et al., 2015).

Excessive weight gain can affect adolescent's physical and emotional well-being. Children in the United States have a shorter life expectancy than their parents do and these comorbidities have been linked to obesity (Felix \& John, 2019). On a BMI-for-age percentile rating, less than the 95th percentile is defined as overweight; a BMI equal to or greater than the 95 th percentile is defined as obese (Felix \& John, 2019). Childhood- and adolescent obesity have adverse effects on blood pressure, insulin, and serum lipid levels. Abnormal levels in these factors can increase chronic disease risk across the lifespan (Felix \& John, 2019).

Traditional counseling by practitioners emphasized increasing the amount of exercise, eating more fruits and vegetables while cutting down on saturated fat, and living a less-sedentary lifestyle. This approach continues today; however, it has not been successful in making lifestyle changes. The National Association of Pediatric Nurse Practitioners and the American Academy of Pediatricians (Felix \& John, 2019) recommended a shift from traditional counseling to a more collaborative, family-centered model that includes motivational interviewing (MI). In this model, the nurse, physician, and family formulate a care plan consistent with the adolescent and closely monitor results (Felix \& John, 2019). MI utilizes a shared, nonjudgmental approach that provides reason and direction for all adolescents (Gruhl \& Van Leuven, 2014). It also focuses on praising 
past, successful health-behavior changes. MI can increase confidence in changing behavior and could also be a favorable predictor of improved, healthier lifestyle outcomes (Felix \& John, 2019).

\section{Evidence-Based Project Purpose}

The purpose of the evidence-based practice (EBP) project was to assess MI interviewing outcomes regarding diet, exercise, and weight loss in adolescents in an underserved community. Pediatric primary care settings in the underserved communities are important areas for improved and effective weight management due to challenges encountered by being overweight or obese. Patient-centered counseling with parent involvement reinforces effective, healthy-behavior changes in adolescents. MI and frequent follow-up can be effective in improving eating behaviors and physical activity in adolescents.

\section{Literature Synthesis}

Studies used to examine this project's evidence were found through a literature search of PubMed and CINHAL. The literature reviews were all randomized controlled trials. All of the evidence suggested a statistically significant, positive effect in patient outcomes by using MI versus the traditional counseling approach. One promising approach for weight-control in adolescents was the utilization of MI in the pediatric primary care setting. MI requires the provider to establish a good rapport with the pediatric population, especially when making healthier behavioral changes in diet and exercise with adolescents and their families. Frequent follow-up visits were needed to build that rapport and to make significant, positive behavior and lifestyle changes. Several randomized controlled trials were implemented to demonstrate more positive outcomes with statistical significance by using MI versus the traditional counseling approach. Pakpour et al. (2014) conducted a randomized control trial of Iranian adolescents 14 
years to18 years of age $(N=357)$ from a local outpatient pediatric clinic in Qazin, Iran. The study's focus was to assess and compare if MI with parent involvement would improve outcomes versus MI with no parental involvement. Adolescent subjects were randomized to one of three groups: MI intervention, MI intervention with parent involvement, or a passive control group. Study measures included height, weight, BMI, body circumferences, and skinfold thicknesses. Psychosocial and behavioral measures were collected initially and at 12 months. The results disclosed significant effects on most outcome measures for MI and MI with parental involvement when compared to those in the passive control group. Motivational interviews with parental involvement revealed a significant clinical change in obesity-related outcomes, more so than MI with adolescents only. One limitation was that the study participants were selected within a Persian culture; therefore, comparisons with other cultures would be necessary. The study concluded that MI with parental involvement effectively changed obesity and overweight outcomes when compared with MI only with adolescents (Pakpour et al., 2014). These findings are noteworthy when implementing MI in pediatric clinics or school settings.

Walpole et al. (2013) conducted a randomized controlled trial consisting of 40 participants ages 10 years to 18 years. Participants were randomly assigned to the control group (i.e., social skills training) or the treatment MI group. Participants $(N=40)$ received 30 minutes of individual therapy every month and included typical diet and exercise counseling. Measured variables included anthropometric measurements and self-efficacy measures. Pre and Post treatment and control groups displayed no significant differences in self-efficacy or height, weight, and BMI outcomes. Except for the MI group, $75 \%$ attended 5 or 6 sessions compared to $40 \%$ attendance in the controlled group. One limitation to the study was the failure to include a second control group of usual care without any counseling. The other limitation to the study 
recognized by the authors was the absence of standard care only, which would have included diet and exercise counseling solely to determine whether participants would have made a difference in BMI and self-efficacy. The study did not display differences between groups; only the MI group attended more sessions. Overall, both groups showed increased self-efficacy and decreased BMI $z$-scores (Walpole et al., 2013).

Freira et al. (2018) conducted a cluster-randomized controlled trial with parallel design in a school setting. The study's purpose was to evaluate a Motivational Interviewing Group (MIG) versus the Conventional Counseling Group (CCG). The study consisted of 800 participants, 100 from 8 different high schools. Both groups had three face-to-face 30-minute interviews 3 months apart; outcomes were BMI $z$-scores, abdominal circumference, percentage of fat and muscle, and blood pressure. MIG participants showed significant improvement in all anthropometric scores at 6 months, and CIG participants did not offer a substantial change in the variables. The three intervention-sessions scoring were statistically significant in MIG $(p<.001)$. Limitations to the study included that the participants were only female, recruited from the urban community, and an absence of a long-term follow-up. Another limitation was the use of BMI $z$-scores instead of BMI (Freira et al., 2018). The overall positive effects in the study with school-based MI suggested that pediatricians or nurse practitioners could play an essential role in preventing pediatric obesity. Schools could serve as a meaningful venue for improving weight reduction. MI counseling significantly and effectively enhanced adolescent's overall health, especially in those adolescents who are overweight.

The evidence reviewed in each study demonstrated the benefits of MI for weight control counseling in adolescents. Although MI has been used to improve diet and physical activity behaviors in the adult population, the evidence for obesity prevention and treatment in children is 
evolving quickly (Felix et al., 2019). Currently, rates of pediatric obesity are increasing, along with an anticipated increase in lifelong chronic disease. Lifestyle changes, MI, and the importance of frequent follow-up with a pediatrician or nurse practitioner should help to improve obesity in the pediatric population.

\section{Evidence-Based Practice Conceptual Framework}

The Iowa Model of Evidence-based Research supported translating the evidence of the project into practice. Utilizing a growth chart, adolescents were identified in one pediatric practice where $85 \%$ of patients were above normal weight. The project's aim was to see if MI interviewing that was focused on nutrition, exercise, and frequent follow-up would decrease the adolescents' weight by $5 \%$ in 4 months. The model suggested forming a team of all pediatric stakeholders: the pediatric clinic stakeholders, including pediatricians, DNP student, nurse practitioner, administrator, front-office and back-office staff; adolescent patients; and families. The team was responsible for developing, implementing, and evaluating the EBP clinical project (Titler et al., 2001). The Iowa Model efficiently guided all stakeholders involved in the clinical project.

\section{Methods}

\section{Practice Setting}

The practice setting for this EBP project was in an underserved rural community in Imperial County. El Centro, CA is near the United States - Mexico border. Childhood and adolescent obesity contribute to health disparities in Imperial County families; the highest rates of overweight and obesity are among those in fifth, seventh, and ninth grades when compared to other counties in California (Ayala et al., 2015). Additional challenges exist for parents of children who are overweight and obese, particularly for underserved communities. The practice 
setting for this project was one pediatric clinic with two advanced nurse practitioners, two pediatricians, and four back-office medical assistants.

\section{Interventions}

A modified clinical screening questionnaire for eating behaviors was implemented over 4 months for pre and post analysis. Health care providers initially screened for overweight and obese adolescents during annual physicals and follow-up appointments. Thirty adolescents at the 85th percentile or above on the growth chart received pre and post screening questionnaires associated with eating behaviors and activity. The screening questionnaire consisted of eight 24hour recall questions including eating habits, restaurant and fast-food consumption, sugar-added beverages, fruit and vegetable servings, daily breakfast, restaurant-size portions, and physical activity. Each item was scored on a 7+ point scale (Figure 1). 


\section{Figure 1}

Screening Questionnaire

\section{Screening Questionnaire for Eating Behaviors in Teens}

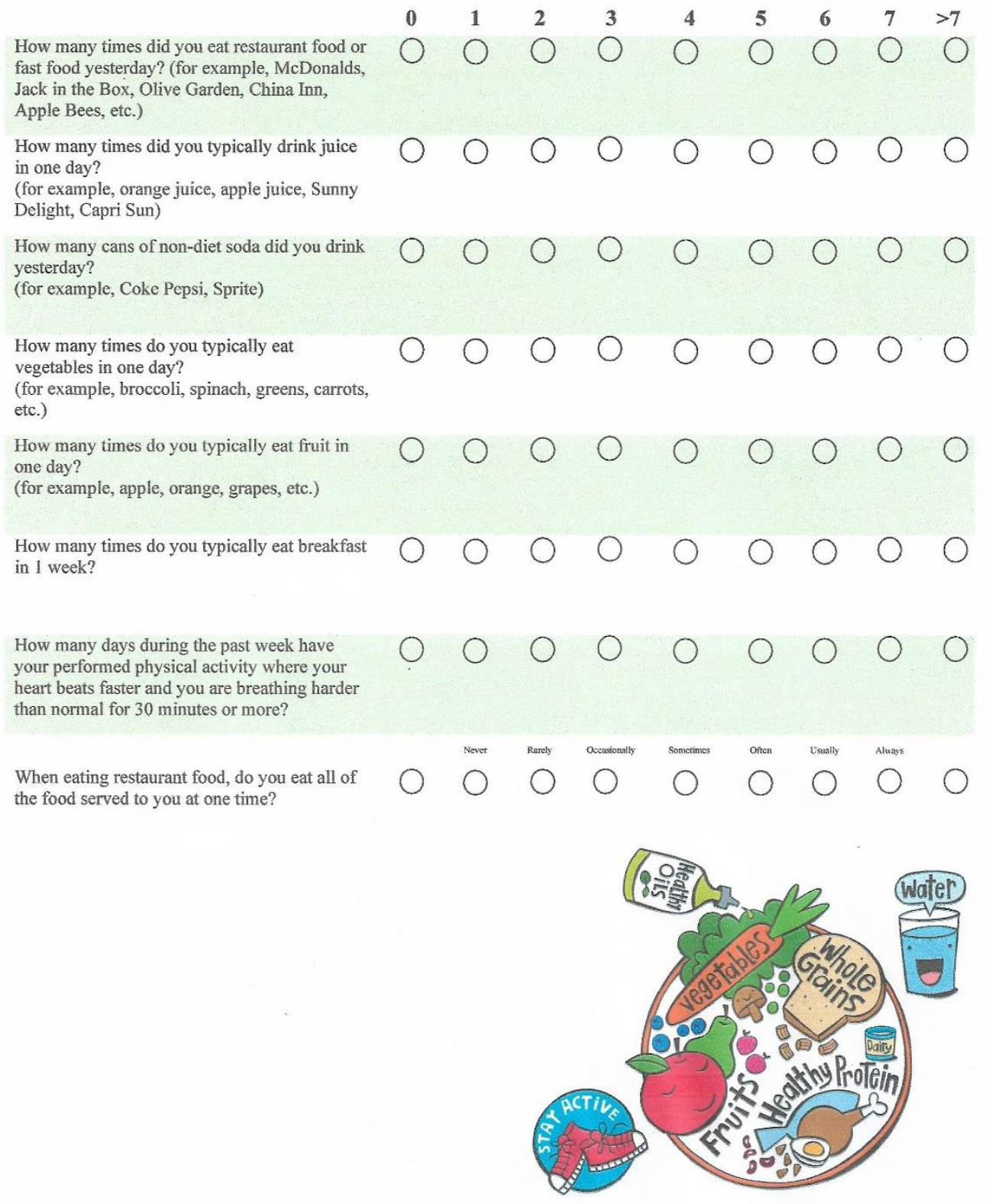

Note. Adapted from "Creating a Clinical Screening Questionnaire for Eating Behaviors Associated with Overweight and Obesity," by J. L. J. Greenwood, M. A. Murtaugh, E. M. Omura, S. C. Alder, and J. B. Stanford, 2008, Journal of the American Board of Family Medicine, 21(6), p. 548 (https://doi.org//10.3122/jabfm.2008.06.070265). Copyright 2008. 
Once the screening tool was completed, the nurse practitioner reviewed responses with parents and adolescents. The nurse practitioner also examined each of the adolescent's body mass index (BMI) and the plotting data on the Centers for Disease Control and Prevention growth charts (United States Preventive Services Task Force, 2017). The nurse practitioner reviewed the prescreening questions and BMI findings with both the adolescent and parent. After reviewing results, the nurse practitioner implemented individualized education with parent involvement and MI with monthly visits. All 30 adolescents had monthly follow-up appointments with weight checks for the next 4 months. The same screening questionnaire was given on the fourth month to evaluate eating behaviors and physical activity changes. The nurse practitioner further evaluated the BMI for any changes in the BMI percentile rank.

\section{Analysis}

Thirty adolescents from the 85 th percentile and above on the BMI growth chart were given the pre and post-screening questionnaire for eating behaviors and physical activity. The screening questionnaire displayed common eating behaviors along with the frequencies of those behaviors and how those behaviors were associated with BMI, overweight and obesity. BMI and weight loss were further evaluated by pre and post questionnaire responses to identify if there was an improvement in healthier eating behaviors and physical activity along with a decrease overall BMI by 5\%. Another measure compared the number of follow-up visits with improved healthy behaviors in post questionnaires.

\section{Ethical Considerations}

This EBP project obtained approval from the University of San Diego Institutional Review Board before launching the project (Appendix A). 


\section{Results}

This project examined a total of 30 adolescent patients at the 85 th percentile or above according to the growth chart on the initial screening. All adolescents were given the pre and post-screening questionnaire for eating behaviors and physical activity. There was a total of four follow-up visits scheduled, one every month for 4 months. Out of the 30 patients in this EBP project, 20 patients were seen in all four visits. All adolescents had the opportunity to be seen at the clinic or via tele-visit due to the COVID-19 pandemic. In the adolescents seen in all four visits, healthier behaviors increased, and BMI decreased by the target goal of 5\% or more ( $r=$ $-.75, p<.001 ;$ Figure 2).

\section{Figure 2}

\section{Comparison of Changes in BMI and Behavior}

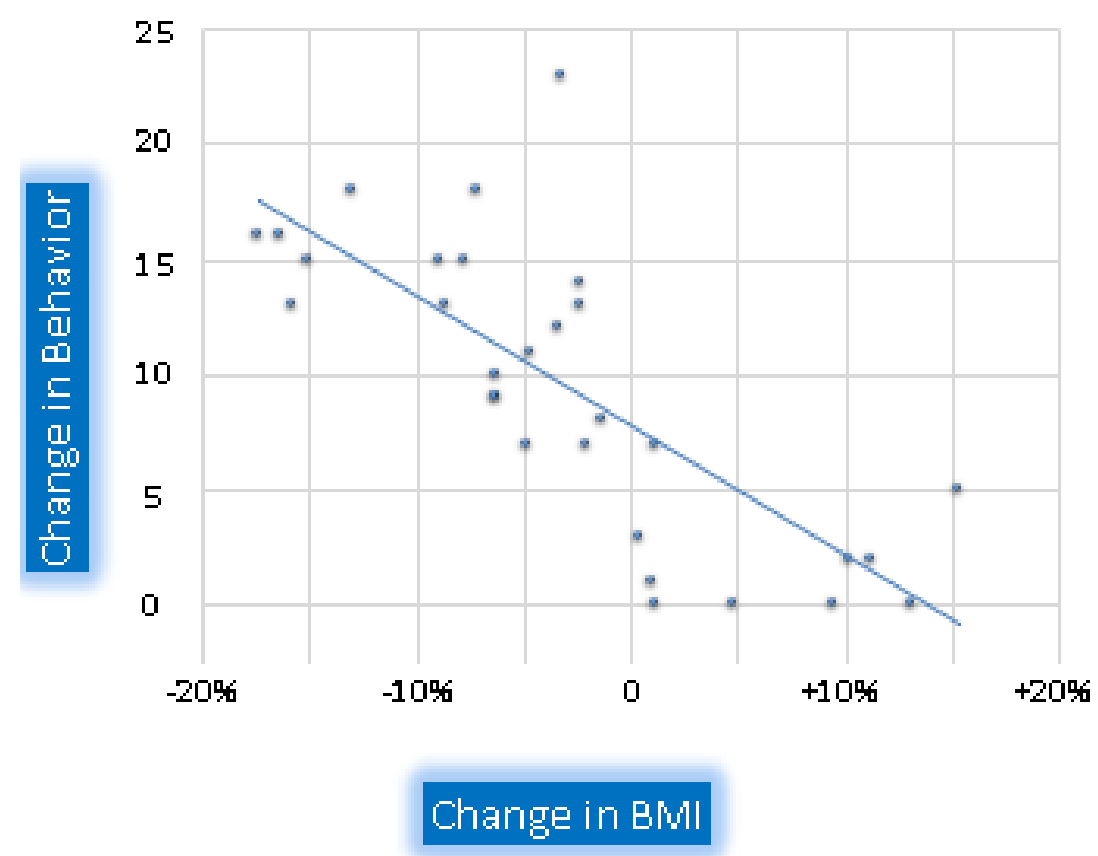

The 10 patients who did not follow up on monthly visits gained $5 \%$ or more at the post-screening visit and eating behaviors stayed the same or worsened. It was evident that, as the number of visits increased, health behaviors also increased $(r=.79, p<.001$; Figure 3$)$. 


\section{Figure 3}

Comparison of Number of Health Care Visits and Change in Behaviors

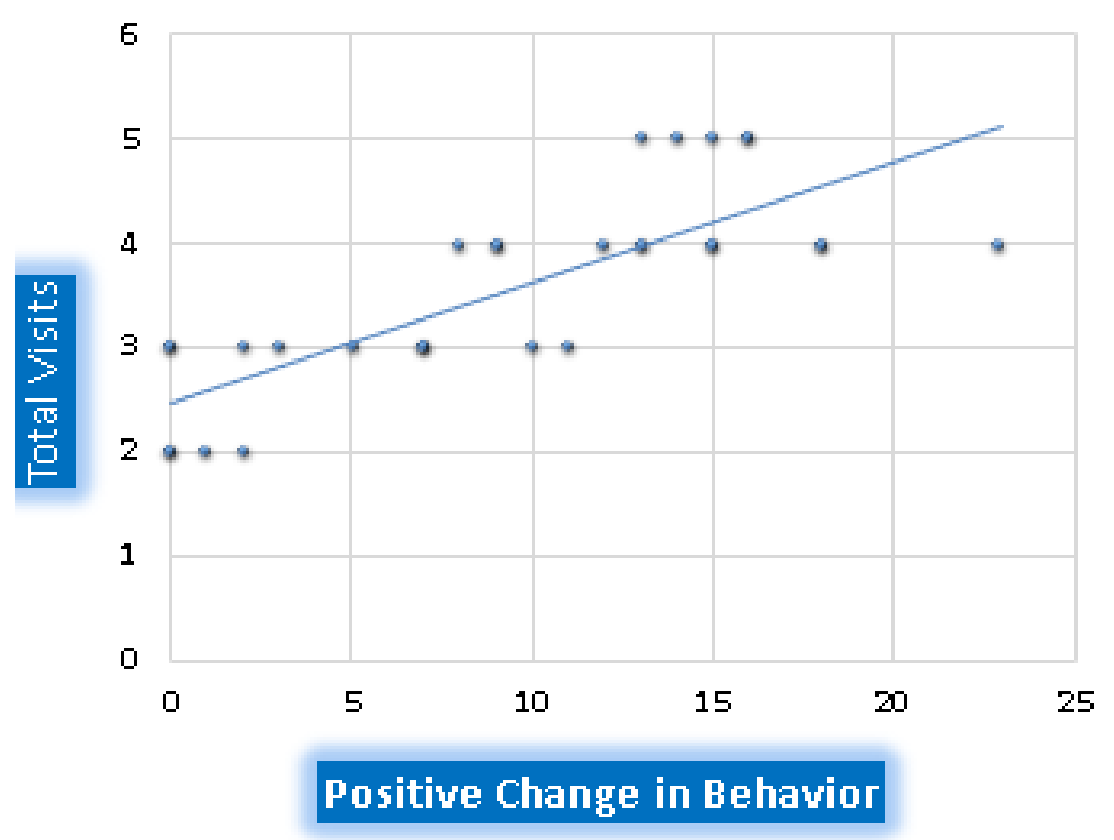

\section{Discussion}

The clinical screening questionnaire adapted and modified for the EBP project included questions specific to "eating behaviors related to overweight and obesity in the adult population" (Greenwood et al., 2008, p. 539). Greenwood et al. (2008) created a screening tool examining specific eating behaviors and how they related to overweight and obesity. Their study focused on particular eating behaviors known to lead to overweight and obesity, such as fast-food eating, large portion sizes, and sugary beverages. Variously, some questions were about positive, healthy eating behaviors such as low energy-dense foods (e.g., fruits, vegetables) and daily breakfast intake. Physical activity was also included in the questionnaire. The goal of utilizing the screening questionnaire in the adolescent patient was to identify potentially-modifiable behaviors that the nurse practitioner could discuss with both the patient and the parents. MI with guided behavioral modifications, identification of healthy and unhealthy responses to the survey, and 
parent involvement were associated with improved diet and physical activity in the adolescent patient.

The U.S. Task Force on Screening for Obesity in Children and Adolescents (2017) reported that early screening with promising behavioral interventions in the primary care setting could lead to improvements in overweight and obesity. Early screening and providers discussing behavioral eating modifications with parental involvement decreased BMI (Freira et al., 2018). Frequent visits also motivated patients to modify their own diets and improve physical activity. The use of MI to collaborate with the adolescent patient would likely exceed a 10-minute visit. However, this investment of additional clinic time could prevent subsequent chronic illnesses in these young adults due to overweight and obesity issues (Gruhl \& Van Leuven, 2014).

The estimated lifetime additional medical cost for an obese child is $\$ 19,000$ and the alternative of a typical adolescent's average weight gain is $\$ 12,660$ (Finkelstein et al., 2014). Early overweight and obesity prevention should be a primary goal in the pediatric clinical setting. The harmful effects of obesity transcend into early adulthood where the differential medical costs are even higher. Therefore, prevention efforts should focus on improving weight management in adolescents (Finkelstein et al., 2014). For the 20 patients with improved weight and eating behaviors during this 4-month EBP project, if sustained, could result in a potential cost savings of $\$ 140,000$. The additional medical cost for the 10 patients who did not make progress in either behavior changes or weight reduction could be estimated at $\$ 50,000$. Obesity prevention methods should be an essential goal for all pediatric primary care settings.

According to the research literature, the use of MI for healthier behavioral changes and weight control would be beneficial for adolescents along with parent involvement. Multiple studies reported significant, positive correlations using MI versus the traditional counseling 
approach. A non-judgmental, empathetic, and shared approach was ideal for adolescents (Gruhl \& Van Leuven, 2014). MI utilization in the pediatric primary care office setting is a promising approach to weight-control and healthy behavioral changes as measured by a decrease in BMI and improved nutritional lifestyle changes. With the current pediatric obesity epidemic, a change from traditional counseling to a more shared family-centered model is much needed.

\section{Limitations}

This practice intervention was not without limitations. One limitation was that the modified screening questionnaire used in the EBP project (Figure 1) had not been previously tested on adolescents; only in the adult population. The questionnaire was created and evaluated for specific eating behaviors and how they were related to overweight and obesity (Greenwood et al., 2008). The original questionnaire was designed to be implemented in primary care clinics to identify and modify eating behaviors, but was not tested on adolescent patients. Although not validated on this EBP project's adolescent population, it identified key eating behaviors and physical activity associated with excessive weight gain. Another limitation to the study was COVID-19: several patients switched to televisits during the pandemic as parents were fearful of bringing their children to the office setting. The appointment compliance was also affected by the pandemic. It is possible that the 10 adolescents who did not comply with appointment follow-ups were fearful of face-to-face visits. Several factors, including the pandemic, influenced treatment adherence in this underserved community. MI appears to be an effective intervention; therefore, rigorous research studies should be done to examine MI in adolescent obesity treatment.

\section{Conclusion}

A modified screening questionnaire for eating behaviors and physical activity in adolescents appeared to be a beneficial screening tools for the pediatric population. Pediatric 
primary care settings could easily adopt this screening tool as it is not cumbersome as well as being easy to follow by adolescents and their parents. The use of MI and frequent follow-up with parent involvement appeared to improve healthy behaviors and physical activity in adolescents. This EBP project aimed to help decrease the lifelong health care costs and economic burden affecting the current pediatric population. Future EBP projects can focus on methods such as televisits and MI on diet and physical activity to increase adherence and retention as well as decrease adolescent overweight and obesity.

\section{Influence on Nursing Practice}

Advanced practice nurses and primary care providers in pediatrics should expand their role in the communities they serve, particularly in the underserved population, to model healthy behaviors. Adolescents and their families could benefit from counseling in obesity prevention. The persistent rise in pediatric obesity suggests a need for differing care approaches that encourage communities to adopt healthier lifestyle changes. Strong parent involvement and culturally competent approaches to care are especially needed for in underserved communities. Clinicians should be mindful of accurate weight assessments in well-child visits and model healthy behaviors. To improve healthy eating and physical environments will take coordination and the assistance of all sectors including health care, schools, government, and the community to ensure today's children lead healthy lives. 


\section{References}

Ayala, G. X., Ibarra, L., Binggeli-Vallarta, A., Moody, J., McKenzie, T. L., Angulo, J., Hoyt, H., Chuang, E., Ganiats, T. G., Gahagan, S., Ji, M., Zive, M., Schmied, E., Arredondo, E. M., \& Elder, J. P. (2015). Our choice/nuestra opción: The Imperial County, California, Childhood Obesity Research Demonstration study (CA-CORD). Childhood Obesity, 11(1), 37-47. https://doi.org/10.1089/chi.2014.0080

Felix, A., \& John, R. M. (2019). Pediatric metabolic syndrome. The Nurse Practitioner, 44(7), 18-25. https://doi:10.1097/01.NPR.0000559841.45754-73

Finkelstein, E. A., Graham, W. C. K., \& Malhotra, R. (2014). Lifetime direct medical costs of childhood obesity. Pediatrics, 133(5), 854-862. https://doi.org/10.1542/peds.2014-0063

Freira, S., Serra-Lemos, M., Fonseca, H., Williams, G., Ribeiro, M., Pena, F., \& do Céu Machado, M. (2018). Anthropometric outcomes of a motivational interviewing schoolbased randomized trial involving adolescents with overweight. European Journal of Pediatrics,177, 1121-1130. https://doi.org/10.1007/s00431-018-3158-2

Greenwood, L. J., Murtaugh, M. A., Omura, E. M., Adler, S. C., \& Stanford, J. B. (2008). Creating a clinical screening questionnaire for eating behaviors associated with overweight and obesity. Journal of American Board of Family Medicine, 21(6), 539-548. https://doi.org/10.3122/jabfm.2008.06.070265

Gruhl, E., \& Van Leuven, K. A. (2014). Motivational interviewing for adolescents: Behavior counseling for diet and exercise. The Journal of Nurse Practitioners, 10 (7): 493-499. http://dx.doi.org/10.1016/j.nurpra.2014.04.006 
Hales, C. M., Carroll, M. D., Fryar, C. D., \& Ogden, C. L. (2017). Prevalence of obesity among adults and youth: United States, 2015-2016 (NCHS Data Brief 288). http://www.cdc.gov/nchs/data/databriefs/db288_table pdf\#3

Pakpour, A. H., Gellert, P., Dombrowski, S. U., \& Fridlund, B. (2015). Motivational interviewing with parents for obesity: An RTC. Pediatrics, 135(3), e644-e652. https://10.1542/peds.2014-1987

Titler, M. G., Kleiber, C., Steelman, V. J., Rakel. B. A., Budreau, G., Everett, L. Q., Buckwalter, K. C., Tripp-Reimer, T., \& Goode, C. J. (2001). The Iowa model of evidence-based practice to promote quality care. Critical Care Nursing Clinics of North America, 13(4), 497-509. https://doi.org/10.1016/S0899-5885(18)30017-0

U.S. Preventive Services Task Force. (2017). Screening for obesity in children and adolescents: US Preventive Services Task Force recommendation statement. Journal of the American Medical Association, 317(23), 2417-2426. https://doi.org/10.1001/jama.2017.6803

Walpole, B., Dettmer, E., Morrongiello, B. A., McCrindle, B. W., \& Hamilton, J. (2013). Motivational interviewing to enhance self-efficacy and promote weight loss in overweight and obese adolescents: A randomized controlled trial. Journal of Pediatric Psychology, 38(9), 944-953. https://doi.org/10.1093/jpepsy/jst023 